\title{
Spain is different. Cobertura en línea de la cumbre del clima de París en cinco países
}

\section{Spain is different. Online coverage of the Paris climate summit in five countries \\ Spain is different. Cobertura on-line da conferência climática de Paris em cinco países}

María Carmen Erviti Ilundáin, Universidad de Navarra, Pamplona, España (mcerviti@unav.es)

Bienvenido León, Universidad de Navarra, Pamplona, España (bleon@unav.es)

\begin{abstract}
RESUMEN | Dada la importancia de los contextos nacionales para adecuar la comunicación del cambio climático a cada país, esta investigación tuvo por objetivo averiguar si la cobertura realizada por los medios españoles es diferente de la de otros países en volumen de artículos, temas y fuentes. Realizamos un análisis de contenido de publicaciones en línea $(\mathrm{n}=527)$ sobre la cumbre del clima de París (COP21, 2015) en 13 medios de cinco países: Estados Unidos y Reino Unido (del bloque anglosajón), y Alemania, Francia y España (de la Europa continental). Los resultados indican que la cobertura realizada en España difiere notablemente de la de los otros países, incluidos los de Europa continental. El alineamiento ideológico de los medios españoles no parece afectar al volumen de cobertura sobre el cambio climático, como ocurre en el ámbito internacional. Estos resultados permiten matizar las conclusiones de investigaciones previas y proponer recomendaciones para la cobertura mediática en España.
\end{abstract}

PALABRAS CLAVE: cambio climático; cobertura informativa; COP21; medios en línea; España. 
ABSTRACT / Given the relevance of national contexts in adapting climate change communication to each country, this research aims to find to which extent the coverage in the Spanish media differs from that of other countries, regarding the volume of articles, topics, and sources. We conducted a content analysis of online publications $(n=527)$ on the Paris climate summit (COP21, 2015) in 13 media outlets from five countries: the United States and the United Kingdom (from the Anglo-Saxon block), and Germany, France, and Spain (from continental Europe). The results indicate that the coverage provided in Spain differs markedly from that of other countries, including those of continental Europe. The ideological alignment of the Spanish media does not seem to affect the volume of coverage regarding climate change, contrary to what happens in the international arena. These results make it possible to nuance the conclusions of previous research and propose several recommendations for climate change coverage in Spain.

KEYWORDS: climate change; media coverage; COP21; online media; Spain.

RESUMO|Dada a importância dos contextos nacionais para adaptar a comunicação sobre as mudanças climáticas a cada país, esta pesquisa pretende saber se a cobertura feita pelos meios de comunicação espanhóis difere da de outros países em volume de artigos, temas e fontes. Realizamos uma análise de conteúdo de publicações online $(n=527)$ na conferência do clima de Paris (COP21, 2015) em 13 veículos de mídia de cinco países: os Estados Unidos e o Reino Unido (do bloco anglo-saxão) e a Alemanha, a França e a Espanha (da Europa continental). Os resultados indicam que a cobertura oferecida na Espanha difere marcadamente da de outros países, incluindo a Europa continental. O alinhamento ideológico da mídia espanhola não parece afetar o volume de cobertura das mudanças climáticas, como acontece no cenário internacional. Esses resultados permitem qualificar as conclusões de pesquisas anteriores e propor recomendações para a cobertura da mídia na Espanha.

PALAVRAS CHAVE: mudança climática; cobertura informativa; COP21; mídia online; Espanha. 


\section{INTRODUCCIÓN}

En los años sesenta del siglo pasado, el Ministerio de Información y Turismo de España lanzó una campaña para atraer a turistas europeos con el eslogan Spain is different. Hasta hoy, la frase sigue utilizándose para justificar diferencias políticas o sociales entre España y otros países de su entorno. En la misma línea, este artículo investiga hasta qué punto la cobertura mediática sobre el cambio climático ${ }^{1}$ realizada por los medios españoles es diferente a la de otros países. Según la Agencia Europea del Medio Ambiente (European Environment Agency, 2017), España es una de las naciones europeas más afectadas por los impactos del cambio climático. Sin embargo, en general, el cambio climático no es un tema muy prominente para los medios de comunicación españoles (Lopera \& Moreno, 2014). Específicamente, el estudio se centra en la cobertura en medios en línea de la cumbre del clima de París (COP21, 2015), considerada la más importante entre las celebradas en la última década.

La globalización ha propiciado un periodismo más desterritorializado, en el que las noticias con contenidos internacionales abundan (Berglez, 2008). El cambio climático es uno de esos temas que se prestan sobremanera a que los medios de comunicación lo enfoquen desde el ámbito internacional. De hecho, hay estudios que indican que la cobertura mediática del cambio climático parece conducirse de una manera relativamente homogénea en todo el mundo, girando episódicamente en torno a eventos como las cumbres del clima y generando subtemas y debates similares en los diferentes países (Painter \& Schäfer, 2018). Sin embargo, los contextos nacionales son importantes, ya que determinan los discursos y las acciones específicas sobre el cambio climático (Harrison \& Sundstrom, 2007), y con frecuencia los periodistas se atienen a esos intereses domésticos para interpretar el tema (Kunelius \& Eide, 2012). Por lo tanto, los estudios comparativos entre países tienen gran interés, dado que ayudan a dar respuestas comunicativas que contribuyen a afrontar el cambio climático considerando las circunstancias de cada territorio.

La revisión más reciente de estos estudios comparativos en este ámbito es la de Painter y Schäfer (2018). Estos autores señalan que diferencias como el volumen de cobertura en cada país y la mayor o menor contestación al consenso científico sobre el cambio climático en la esfera pública nacional configuran tres bloques

1. Cambio climático fue definido por las Naciones Unidas como un cambio del clima de la Tierra atribuido, directa o indirectamente, a la actividad humana (CMNUCC, 1992). Otra expresión que remite a este fenómeno es calentamiento global, que indica el incremento de la temperatura media del planeta, considerada como la huella del cambio climático (Wigley, 1999). 
de países respecto de la comunicación del cambio climático: anglosajón, Europa continental y Sur Global ${ }^{2}$. Los países anglosajones otorgan una mayor cobertura al cambio climático y muestran un mayor nivel de debate, seguidos por el bloque de la Europa Continental y por los países del Sur.

En este artículo se investiga hasta qué punto la cobertura mediática sobre el cambio climático que se realiza en España difiere de la de otros países del bloque de la Europa continental y del anglosajón. El estudio está justificado puesto que, según la Agencia Europea del Medio Ambiente (European Environment Agency, 2017), España es uno de los países europeos más afectados por los impactos del cambio climático. Se considera necesario conocer comparativamente cómo se está llevando a cabo la cobertura mediática de este importante reto para proponer las recomendaciones oportunas. Por ello, analizamos tres aspectos clave de la comunicación del cambio climático en medios: volumen de cobertura, temática y fuentes empleadas.

\section{Volumen de cobertura}

El volumen de la cobertura informativa ha sido uno de los aspectos más frecuentemente estudiados. Como se observa en el gráfico 1 a partir de la base de datos abierta Media and Climate Change Observatory de la Universidad de Colorado, desde una perspectiva geográfica tanto los medios anglosajones como europeos ofrecen una cantidad de información significativamente mayor que los medios del Sur.

En el caso de los países de la esfera anglosajona, los intereses económicos y políticos confluyen para propiciar una cobertura periodística en la que las posturas escépticas y negacionistas adquieren mayor protagonismo que en el resto de los países. En Estados Unidos, numerosos autores mencionan el éxito de la industria de los combustibles fósiles en generar dudas sobre el cambio climático (Zehr, 2000; Corbett \& Durfee, 2004; McCright \& Dunlap, 2003). Otros estudios apuntan a la polarización política de los periódicos del Reino Unido ante el cambio climático (Carvalho, 2005, 2007; Lockwood, 2009). En este sentido, la mayor cobertura periodística del cambio climático se corresponde con el hecho de que en los países anglosajones es un tema controvertido, lo cual añade valor noticioso.

Mientras en el ámbito anglosajón la controversia es un elemento importante, en los países de la Europa continental el cambio climático no llega a ser un tema político tan polarizado (Weart, 2021). Alemania destaca por su alto grado de consenso y compromiso (Gavin, 2009), de tal modo que los medios de comunicación ignoran las incertidumbres y ponen el foco en la necesidad de una acción inmediata contra el cambio climático (Weingart, Engels, \& Pansegrau, 2000).

2. Sur global es un término para referirse al tercer mundo o al conjunto de países en vías de desarrollo. 
1,500

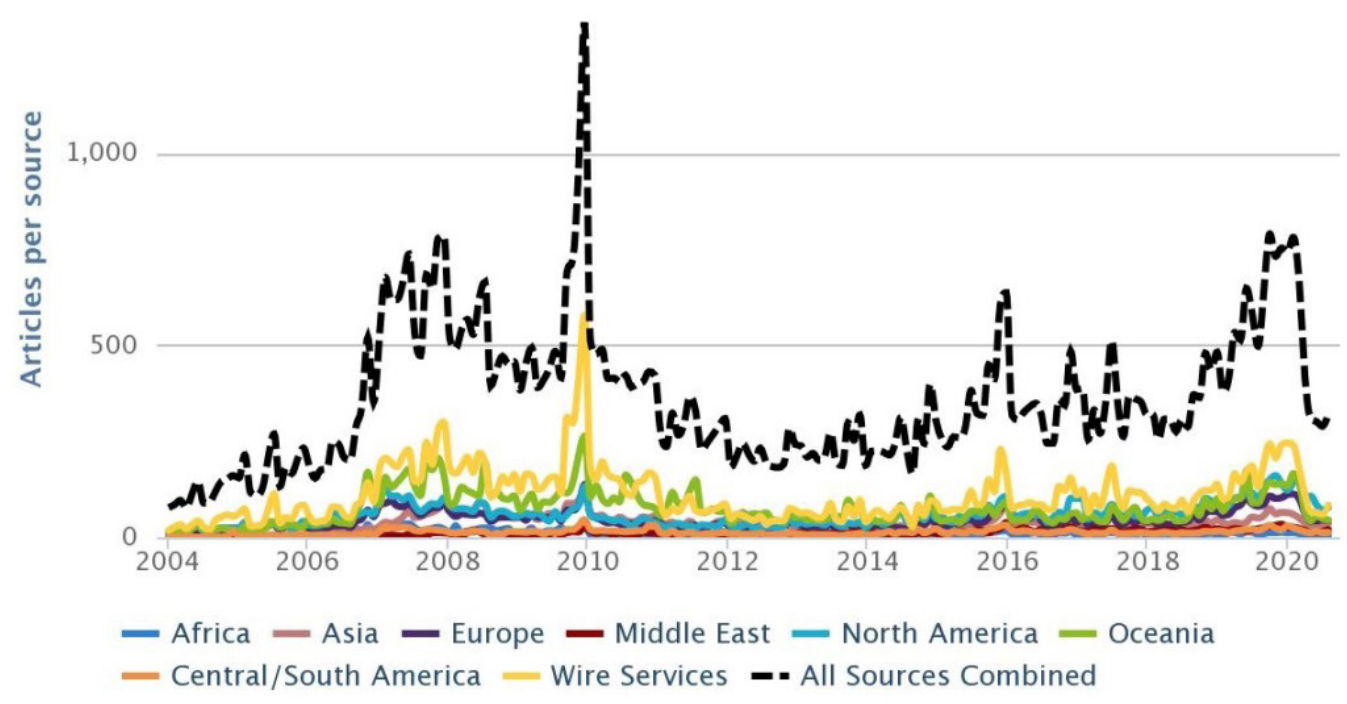

Gráfico 1. Cobertura mundial de prensa del cambio climático 2004-2020

Fuente: Media and Climate Change Observatory, University of Colorado (2021).

Los países nórdicos también sobresalen al situarse en la primera línea de las políticas pro-Kioto, y la cobertura de sus medios de comunicación refleja ese posicionamiento (Berglez, Höije, \& Olausson, 2009). Estas dinámicas del consenso parecen ir asociadas a un volumen de cobertura inferior al que se produce cuando hay disenso.

Además, un estudio comparativo más reciente vincula la atención mediática al cambio climático con los contextos legislativos y económicos de cada país. Un mayor desarrollo de las regulaciones en este ámbito se corresponde con una mayor cobertura. Por el contrario, los países con mayores tasas de desempleo le otorgan menos cobertura (Barkemeyer, Figge, Hoepner, Holt, Kraak, \& Yu, 2017).

Por otra parte, trabajos que han estudiado el rol ideológico de los medios de comunicación muestran que el volumen de cobertura del cambio climático era mayor en los medios de comunicación de izquierdas que en los conservadores (Carvalho, 2007; Dirikx \& Gelders, 2010; Dotson, Jacobson, Kaid, \& Carlton, 2012).

Respecto de la atención mediática en España, el Media Climate Change Observatory (MeCCO), liderado por la Universidad de Colorado ${ }^{3}$, contabiliza la cobertura del cambio climático en los tres periódicos españoles de mayor

3. MeCCO es una colaboración entre universidades que monitoriza 117 medios (periódicos, radios y televisiones) en 53 países de los diferentes continentes. Los criterios de selección de medios responden a la diversidad geográfica, el volumen de circulación y el acceso a los archivos. 
circulación (El País, El Mundo y La Vanguardia). Por este seguimiento, sabemos que los picos de atención mediática son similares a los de otros países (Brossard, Shanahan, \& McComas, 2004; Schmidt, Ivanova, \& Schäfer, 2013). FernándezReyes, Raigada y Águila Coghlan (2017) comparan sus resultados de cobertura en España con los de otros estudios en el ámbito internacional y concluyen que existe un paralelismo en el desarrollo de la cobertura a lo largo del tiempo, de tal modo que los picos coinciden, a nivel internacional, con la publicación de los informes del IPCC y las cumbres del clima más destacadas.

En comparación con otros países, España está por debajo del promedio en cobertura del cambio climático (Schmidt et al., 2013). Según la investigación realizada, en 2005, 2006 y 2011 este era un tema marginal. Solo 0,2\% de todas las informaciones en los principales periódicos nacionales y 0,19\% de las noticias de los canales nacionales se centraron en el cambio climático en esos años (León, 2014).

\section{Encuadres temáticos}

Como se vio, las diferencias en el volumen de cobertura mediática entre países en torno al cambio climático se relacionan con los encuadres temáticos, ya sea de incertidumbre y controversia o de certidumbre y consenso. Nos referimos también a otros temas habituales en la cobertura informativa.

En muchos países, las informaciones sobre el cambio climático con frecuencia se enmarcan como un tema político que, además de estar presente en el ámbito internacional, recala en el debate nacional (Carvalho, 2007; Boykoff \& Boykoff, 2007; Corbett, Young, \& Davis, 2009). En España, el enfoque político también es el predominante. Esto se debe a que el grueso de la información sobre el cambio climático se publica durante el periodo de las cumbres, en las que las negociaciones políticas internacionales desempeñan un rol fundamental (León \& de Lara, 2013; Blanco-Castilla, Quesada, \& Teruel Rodríguez, 2013). Sin embargo, la investigación realizada en España no contaba con un análisis específico de temáticas.

Los encuadres temáticos del cambio climático en la esfera internacional han sido analizados principalmente por James Painter, que se toma como autor de referencia para el análisis de temas presentado en este artículo. Sus sucesivos trabajos consideran el encuadre del escepticismo (Painter \& Ashe, 2012), las temáticas del riesgo y la incertidumbre (Painter, 2013), así como las del desastre o catástrofe y oportunidad (Painter, 2017). Frente al predomino del encuadre de desastre o catástrofe, es decir, la presentación de los impactos adversos del cambio climático, el encuadre de la oportunidad, entendido como el avance hacia un modelo económico y social más sostenible, ha ido ganando terreno (Painter, 2017). Estos estudios, con muestras de diferentes países, no son concluyentes respecto del rol de la ideología de los medios en relación con los temas predominantes. 


\section{Fuentes}

En las últimas décadas, se ha consolidado una serie de fuentes de referencia sobre el cambio climático para los medios de comunicación. Algunas tienen visibilidad a escala internacional: participantes en las cumbres del clima (políticos, representantes de ONG, etc.), expertos del IPCC e incluso las llamadas celebrities (Anderson, 2011). Otras fuentes políticas y de la sociedad civil tienen alcance nacional o local. Estudios previos muestran una prominencia a nivel internacional de las voces políticas y gubernamentales (Eide \& Kunelius, 2010; Comfort, Tandoc, \& Gruszczynski, 2020). También las noticias en línea apuestan por las fuentes oficiales, incluso con mayor frecuencia que las televisiones o los periódicos (Anderson, 2015). Los medios de comunicación españoles repiten este patrón: los políticos son las voces que prevalecen en las informaciones sobre el cambio climático (Piñuel, 2013).

La norma periodística de proporcionar un equilibrio entre diferentes puntos de vista ha provocado que las fuentes escépticas tengan una fuerte representación en los medios de comunicación de algunos países, principalmente los anglosajones (Boykoff \& Boykoff, 2007). Petersen, Vincent y Westerling (2019) descubrieron que 386 voces escépticas sobre el cambio climático, incluyendo académicos, científicos, políticos y empresarios, tuvieron más visibilidad en la prensa internacional que los 386 científicos del clima más reconocidos. Aunque esta mayor visibilidad de las fuentes escépticas se producía incluso en medios orientados ideológicamente hacia la izquierda, un análisis de periódicos de Reino Unido, Alemania y Suiza, llevado a cabo por Schmid-Petri (2017), concluyó que determinados medios conservadores mostraban más apertura a este tipo de voces. Sin embargo, en España no se ha detectado que las fuentes escépticas sean relevantes (de Lara, 2013).

\section{PREGUNTAS DE INVESTIGACIÓN Y METODOLOGÍA}

Con el objetivo de conocer en qué medida España es un país diferente en su cobertura informativa del cambio climático en medios en línea (tradicionales y nativos), se plantean las siguientes preguntas de investigación:

P1. ¿Es el volumen de cobertura en España similar al de los otros países?

P2. ¿Los encuadres temáticos dominantes sobre el cambio climático en los medios españoles son semejantes a los de otros países?

P3. ¿Las fuentes citadas en los artículos españoles son similares a las citadas en otros países? 
La muestra y los datos empleados se obtuvieron de un estudio previo sobre la cobertura internacional de la $21^{a}$ Conferencia de las Naciones Unidas sobre el cambio climático (COP21) celebrada en París en 2015 (Painter et al., 2017). Esta cumbre marcó un hito en la lucha contra el cambio climático, ya que la comunidad internacional alcanzó un acuerdo para reducir las emisiones de gases de efecto invernadero a partir de 2020. El anterior acuerdo de relevancia, el Protocolo de Kioto, se produjo en 1997, lo cual muestra las dificultades de la negociación sobre el clima.

La COP21 de París atrajo un alto nivel de atención mediática. Un estudio comparativo de su cobertura en periódicos de 50 países indica que el volumen de cobertura del cambio climático durante esta reunión fue el más alto desde 2004, a excepción de la cumbre de Copenhague, en 2009. En España también marcó un pico en la cobertura mediática del cambio climático (Fernández-Reyes, 2016). Por lo tanto, la muestra sigue siendo relevante, a pesar de su relativa antigüedad, debido a la impronta del evento y al interés suscitado.

Los países seleccionados para el estudio fueron cinco, dos anglófonos (Estados Unidos y Reino Unido) y tres de la Europa occidental (España, Francia y Alemania), siguiendo a dos de los bloques indicados por Painter y Schäfer (2018) para la comunicación del cambio climático: el bloque anglosajón y el europeo continental. Por cada país se seleccionaron periódicos de referencia para las corrientes ideológicas más representativas de la derecha e izquierda y tres medios nativos digitales: las ediciones en línea de US Today y The New York Times (Estados Unidos); The Telegraph y The Guardian (Reino Unido); Le Figaro y LeMonde (Francia); Frankfurter Allgemeine Zeitung y Süddeutsche Zeitung (Alemania). En España, se seleccionaron El País y El Mundo, de acuerdo con los criterios de difusión y representación ideológica. El País tiene un alcance semanal de $27 \%$ de los usuarios de información en línea y El Mundo llega al 22\% (Negredo, 2018). Además, El País se sitúa en una posición política de centro-izquierda o izquierda, mientras que El Mundo se define como un diario liberal y próximo a las posiciones de la derecha.

El criterio de inclusión en la muestra de medios nativos digitales fue que el primer estudio (Painter et al., 2017) contrastó la cobertura de estos con la de los periódicos tradicionales en sus ediciones digitales. Para la selección de los nuevos medios, se buscaron ejemplos destacados, con presencia en todos los países del estudio ${ }^{4}$, que tienen un interés especial porque incorporan formatos novedosos y otorgan cierta prioridad editorial a los asuntos ambientales. Estos medios son Huffington Post (plataforma de noticias y blogs de opinión de Estados Unidos), Vice

4. La presencia de estos medios en todos los países del estudio se debe a que uno de los objetivos del trabajo previo fue comparar cómo funcionaban las respectivas ediciones nacionales. 
(web que tiene su origen en la revista canadiense del mismo nombre) y Buzzfeed (medio en línea estadounidense centrado en contenidos virales).

En 2015, año de la cumbre del clima de París, ocho de cada diez ciudadanos españoles se conectaban a diario a Internet y los medios de noticias digitales gozaban de un alto nivel de credibilidad (IAB España, 2015). Algunos de los nuevos medios adquirieron relevancia en un corto período de tiempo, pero tuvieron problemas para mantenerse. Por ejemplo, El Confidencial, El Español, OKDiario y Huffington Post se encontraban entre los 15 sitios web más importantes en España, pero las versiones en línea de diarios como El País y El Mundo encabezaban la lista de las webs más leídas (Comscore, 2015). La audiencia de Huffington Post (1\%), Vice, (2\%) y Buzzfeed (2\%) es muy inferior a la de los periódicos españoles de referencia (Negredo, 2018). A comienzos de 2019, algunos de los medios nativos digitales de mayor audiencia llevaron a cabo importantes recortes en sus redacciones. Incluso Buzzfeed -incluido en el este estudio - cerró su delegación en España y en otros países (Rubio, 2019).

El período de muestreo fue de tres semanas (miércoles 25 de noviembre a miércoles 16 de diciembre de 2015), durante el cual se realizó una búsqueda diaria de artículos en los medios web seleccionados. Incluyó todos los días de la cumbre, además de los cinco anteriores y los cinco posteriores. Los términos de búsqueda empleados fueron "calentamiento global” o "cambio climático", y "París” o “COP21", así como sus traducciones en inglés, francés y alemán. La unidad básica de análisis fue el "artículo". Para el objetivo de esta investigación, se define como un contenido en línea que puede presentarse en forma de texto, vídeo, imágenes u otros formatos, normalmente agrupados en torno a un titular (Painter et al., 2017).

Se obtuvo una cantidad de 1883 artículos en los cinco países estudiados, empleados en el análisis del volumen de cobertura (tabla 2). De estos, 1237 artículos $(65,69 \%)$ se publicaron en la prensa en línea de referencia y $646(34,3 \%)$, en los medios nativos digitales.

Posteriormente, se seleccionó una muestra aleatoria de 27,99\% del total de los artículos $(\mathrm{n}=527)$, con el fin de hacer viable un análisis de contenido cuantitativo5: 259 artículos publicados en medios nativos digitales (un 40\% del total de los

5. El análisis de los contenidos de la comunicación es una metodología que parte del principio de que examinando textos es posible conocer no solo su significado, sino también información sobre cómo han sido producidos y sobre las características de sus emisores. Con esta técnica, se consigue la descripción objetiva, sistemática y cuantitativa de la muestra de estudio (Berelson, 1952). 
publicados por estos medios) y 268 artículos publicados en los periódicos tradicionales (21,66\%). Los artículos de Vice y Buzzfeed se incluyeron en la muestra en su totalidad, por contar con un número reducido de publicaciones en todos los países y ser objeto de estudio para el proyecto que encuadra este trabajo. En los países en que también Huffington Post había sumado pocas publicaciones (España y Alemania), se incorporaron en su totalidad a la muestra para poder establecer las oportunas comparaciones (tabla 1). En consecuencia, el análisis posterior de los datos obtenidos se validó teniendo en cuenta porcentajes.

La codificación se llevó a cabo, de forma independiente, por dos codificadores, tras un proceso de formación preliminar en el que se debatió sobre el manual de codificación en detalle para establecer criterios homogéneos. Finalmente, se siguieron los siguientes criterios:

Temas. Siguiendo a Painter (2013), se identificaron varios temas dominantes, definidos como sigue:

- Negociaciones. Incluye explícitamente los detalles del proceso y el progreso de las negociaciones o su resultado. Por ejemplo, la discusión del texto del acuerdo, las distintas posiciones de los países participantes, los compromisos para reducir emisiones, etc.

- Contexto científico. Son las referencias específicas a la ciencia del clima que respalda la necesidad de un acuerdo internacional (por ejemplo, para mantener el calentamiento global por debajo de los 2 grados Celsius). Entre los indicadores están los informes científicos de organismos como el IPCC, o informes de la Agencia Internacional de la Energía y el Banco Mundial, que mencionan el contexto científico.

- Desastre/catástrofe. Consiste en descripciones o afirmaciones de posibles impactos o efectos negativos (a menudo dramáticos) del calentamiento global. Por ejemplo, subida del nivel del mar, episodios de clima extremo como inundaciones o sequías, escasez de alimentos, movimientos de población, daño a los arrecifes de coral, reducción de las cubiertas de hielo, etc.

- Incertidumbre. En este tema se incluyen i) afirmaciones o descripciones relacionadas con las faltas de certeza sobre distintos aspectos de las ciencias del clima, incluyendo rangos de proyecciones sobre aumentos de temperaturas, subida del nivel del mar, ubicación o gravedad de los impactos o falta de fiabilidad de los modelos matemáticos, y ii) la presencia de escepticismo, en sus distintas variantes (de impacto, de atribución, de tendencia o político). 
Países

Medios

Tamaño de la muestra

\begin{tabular}{|c|c|c|}
\hline \multirow{5}{*}{ EE.UU. $n=125$} & New York Times (izquierda) & 22 \\
\hline & USA Today (derecha) & 20 \\
\hline & Huffington Post & 23 \\
\hline & BuzzFeed & 28 \\
\hline & Vice & 32 \\
\hline \multirow{5}{*}{ Reino Unido $\mathrm{n}=116$} & The Guardian (izquierda) & 37 \\
\hline & The Telegraph (derecha) & 16 \\
\hline & Huffington Post & 25 \\
\hline & BuzzFeed & 24 \\
\hline & Vice & 14 \\
\hline \multirow{5}{*}{ Francia $\mathrm{n}=110$} & Le Monde (izquierda) & 30 \\
\hline & Le Figaro (derecha) & 30 \\
\hline & Huffington Post & 30 \\
\hline & BuzzFeed & 10 \\
\hline & Vice & 10 \\
\hline \multirow{5}{*}{ Alemania $\mathrm{n}=81$} & Süddeutsche Zeitung (izquierda) & 31 \\
\hline & Frankfurter Allgemeine Zeitung (derecha) & 31 \\
\hline & Huffington Post & 14 \\
\hline & BuzzFeed & 0 \\
\hline & Vice & 5 \\
\hline \multirow{5}{*}{ España $n=95$} & El País (izquierda) & 26 \\
\hline & El Mundo (derecha) & 25 \\
\hline & Huffington Post & 37 \\
\hline & BuzzFeed & 0 \\
\hline & Vice & 7 \\
\hline & Total & 527 \\
\hline
\end{tabular}

Tabla 1. Muestra para el análisis de contenido, por medios y países

Fuente: Elaboración propia.

- Oportunidad. Contenidos que se refieren a las ventajas de tomar medidas para reducir los riesgos derivados de las emisiones de gases de efecto invernadero, tales como las ventajas de avanzar hacia una economía baja en carbono, oportunidades de negocio, beneficios para la salud, seguridad energética, etc. 
- Justicia climática. Referencias explícitas a la diferente responsabilidad histórica entre países en las emisiones de gases y las distintas cargas en cuanto a mitigación, adaptación o alivio de las consecuencias del cambio climático. También incorpora las menciones a cualquiera de los muchos asuntos éticos que rodean al cambio climático, incluyendo la responsabilidad de la actual generación respecto de futuras generaciones.

- Fuentes. Se codificaron las fuentes en las que se menciona un nombre en específico (o un cargo; por ejemplo, "el primer ministro dijo"), citadas directa o indirectamente. Por lo tanto, no se codificaron las referencias genéricas de instituciones, cuando no se nombra al portavoz, autor o representante. Las menciones de redactores en plantilla de un medio de comunicación no se codificaron. Las fuentes se clasificaron en las siguientes categorías: político del país del reportero; político de otro país; representante de UNFCC (Convención Marco de las Naciones Unidas sobre el Cambio Climático); representante de otro organismo internacional (IPCC, otro organismo de la ONU, etc.); científico o representante de centro de investigación; representante de ONG, think tank, lobby o gabinete estratégico; hombre de negocios; ciudadano de a pie; líder religioso, y otro.

La fiabilidad de los datos recogidos por los codificadores se midió aplicando el coeficiente de Lotus, desarrollado por Fretwurst en $2015^{6}$. Los resultados para este estudio se sitúan por encima de 0,9 (90\% de acuerdo).

\section{RESULTADOS}

\section{Volumen de cobertura}

Los datos del volumen de cobertura ponen de manifiesto que, durante el período de estudio, los medios españoles analizados publicaron un total de 242 artículos. Esto significa que la cumbre atrajo menos atención mediática que en otros países, ya que solo los medios alemanes publicaron menos artículos (152) que los españoles (tabla 2).

Por una parte, en efecto, se comprueba que los países anglosajones incluidos en nuestro estudio (Estados Unidos y Reino Unido), realizaron una cobertura mucho más extensa que la de los países del bloque de Europa continental, excepto Francia. La extensa cobertura francesa está relacionada con el hecho de que la COP21 tuvo lugar en París, lo que significó un interés mediático añadido, debido al factor de la proximidad. Sin embargo, también existen notorias diferencias entre el volumen de cobertura realizado por los otros dos países de la Europa continental -Alemania (152) y España (242) -. 


\begin{tabular}{|c|c|c|c|}
\hline País & Medio & $\mathrm{N}^{\circ}$ artículos & Porcentaje \\
\hline \multirow{5}{*}{ EE. UU. $n=422$} & New York Times & 110 & 5,84 \\
\hline & USA Today & 21 & 1,12 \\
\hline & Huffington Post & 230 & 12,21 \\
\hline & BuzzFeed & 28 & 1,49 \\
\hline & Vice & 33 & 1,75 \\
\hline \multirow{5}{*}{ Reino Unido $n=537$} & The Guardian & 361 & 19,17 \\
\hline & The Telegraph & 63 & 3,35 \\
\hline & Huffington Post & 75 & 3,98 \\
\hline & BuzzFeed & 24 & 1,27 \\
\hline & Vice & 14 & 0,74 \\
\hline \multirow{5}{*}{ Francia $n=530$} & Le Monde & 202 & 10,73 \\
\hline & Le Figaro & 149 & 7,91 \\
\hline & Huffington Post & 157 & 8,34 \\
\hline & BuzzFeed & 12 & 0,64 \\
\hline & Vice & 10 & 0,53 \\
\hline \multirow{5}{*}{ Alemania $n=152$} & $S Z$ & 65 & 3,45 \\
\hline & FAZ & 68 & 3,61 \\
\hline & Huffington Post & 14 & 0,74 \\
\hline & BuzzFeed & 0 & 0,00 \\
\hline & Vice & 5 & 0,27 \\
\hline \multirow{5}{*}{ España n=242 } & El País & 103 & 5,47 \\
\hline & El Mundo & 95 & 5,05 \\
\hline & Huffington Post & 37 & 1,96 \\
\hline & BuzzFeed & 0 & 0,00 \\
\hline & Vice & 7 & 0,37 \\
\hline \multicolumn{2}{|c|}{ Total } & 1883 & 100 \\
\hline
\end{tabular}

Tabla 2. Volumen de cobertura en los medios seleccionados

Fuente: Elaboración propia.

En España, al igual que en la mayoría de los otros países, los medios nativos digitales publicaron menos artículos que los medios tradicionales. Huffington Post es la excepción, ya que en Estados Unidos publicó más artículos que cualquiera de los otros medios, y en Reino Unido y Francia se situó ligeramente por encima del 
medio tradicional con menor volumen de cobertura. Además, en España, los medios nativos digitales no solo publicaron menos artículos que los medios tradicionales, sino que su volumen de cobertura fue notablemente inferior al de sus ediciones en los otros países (con la única excepción de Alemania, donde la cobertura fue todavía inferior a la de España).

También cabe destacar que, en España, El País, medio tradicional alineado políticamente a la izquierda, publicó un número mayor de artículos que su competidor alineado a la derecha (El Mundo). Esta tendencia se repite en la mayoría de los otros países. Sin embargo, en España y Alemania la diferencia entre el número de artículos publicados por el medio de izquierdas y el de derechas es muy pequeña. En España, El País publicó solo ocho artículos más que El Mundo, mientras que en Alemania el medio alineado a la derecha (Frankfurter Allgemeine Zeitung) publicó incluso tres artículos más que su competidor de izquierdas (Süddeutsche Zeitung).

\section{Temas}

En cuanto a los temas principales de los artículos, las negociaciones de la cumbre de París atrajeron en mayor medida la atención de los medios, mientras que las incertidumbres fueron el tema con menos notoriedad. La cobertura realizada por los medios españoles fue relativamente similar a la de los otros países, con la notable excepción de la justicia climática, que tuvo una representación más escasa (tabla 3). Además, cabe destacar que este tema fue abordado por todos los medios españoles en menor medida que sus equivalentes en otros países, independientemente de su tendencia política o trayectoria empresarial.

El segundo tema en el que hay mayor diferencia entre España y la media del resto de los países es oportunidad. En este caso, al contrario que en justicia climática, los porcentajes de los medios españoles son unas veces superiores y en otras inferiores a los de los medios equivalentes en los otros países. Aunque los medios tradicionales y Vice otorgaron mayor peso a este tema, la menor frecuencia con la que aparece en el Huffington Post reduce la diferencia para el conjunto del país.

El medio que prestó mayor atención a las oportunidades del cambio climático fue El País (54\%), con un porcentaje solo superado, entre los medios tradicionales de izquierdas, por The Guardian (68\%). Además, solo en el caso de España, el periódico El País ha publicado menos artículos sobre desastre/catástrofe que su competidor de centroderecha. En los otros cuatro países, por el contrario, el cambio climático como catástrofe es más recurrente en los periódicos de ideología progresista. Otro dato reseñable es que la edición en Alemania de Huffington Post destaca en los temas desastre/catástrofe (64\%) y oportunidad (86\%) por encima de todos los demás medios de la muestra. 


\section{Medio Francia Alemania $\begin{aligned} & \text { Reino } \\ & \text { Unido }\end{aligned}$ EE.UU. España Diferencia}

\begin{tabular}{|c|c|c|c|c|c|c|c|}
\hline \multirow{6}{*}{ 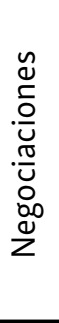 } & Periódico dcha. & 37 & 81 & 88 & 35 & 36 & -24 \\
\hline & Periódico izda. & 53 & 61 & 73 & 41 & 77 & 20 \\
\hline & Huffington Post & 50 & 79 & 60 & 13 & 62 & 12 \\
\hline & Vice & 40 & 20 & 43 & 34 & 43 & 9 \\
\hline & BuzzFeed & 0 & & 50 & 39 & & -22 \\
\hline & Media por país & 42 & 69 & 64 & 33 & 58 & 6 \\
\hline \multirow{6}{*}{ 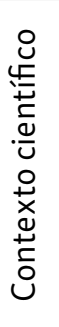 } & Periódico dcha. & 23 & 55 & 56 & 40 & 52 & 9 \\
\hline & Periódico izda. & 23 & 35 & 38 & 41 & 46 & 12 \\
\hline & Huffington Post & 17 & 29 & 40 & 52 & 27 & -8 \\
\hline & Vice & 0 & 40 & 36 & 50 & 57 & 26 \\
\hline & BuzzFeed & 0 & & 42 & 36 & & -20 \\
\hline & Media por país & 17 & 42 & 42 & 44 & 41 & 5 \\
\hline \multirow{6}{*}{ 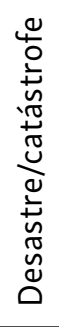 } & Periódico dcha. & 27 & 35 & 50 & 45 & 44 & 5 \\
\hline & Periódico izda. & 27 & 45 & 57 & 55 & 38 & -8 \\
\hline & Huffington Post & 33 & 64 & 44 & 52 & 49 & 1 \\
\hline & Vice & 20 & 60 & 50 & 44 & 43 & -1 \\
\hline & BuzzFeed & 10 & & 46 & 50 & & -27 \\
\hline & Media por país & 26 & 46 & 50 & 49 & 44 & 1 \\
\hline \multirow{6}{*}{ 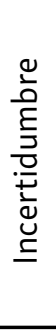 } & Periódico dcha. & 17 & 19 & 31 & 40 & 12 & -15 \\
\hline & Periódico izda. & 10 & 13 & 16 & 23 & 12 & -4 \\
\hline & Huffington Post & 3 & 7 & 0 & 22 & 11 & 3 \\
\hline & Vice & 0 & 40 & 7 & 13 & 43 & 28 \\
\hline & BuzzFeed & 10 & & 8 & 11 & & -7 \\
\hline & Media por país & 9 & 16 & 12 & 20 & 14 & 0 \\
\hline \multirow{6}{*}{ 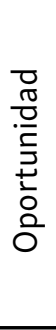 } & Periódico dcha. & 30 & 42 & 50 & 35 & 48 & 9 \\
\hline & Periódico izda. & 23 & 35 & 68 & 14 & 54 & 19 \\
\hline & Huffington Post & 20 & 86 & 76 & 52 & 35 & -24 \\
\hline & Vice & 20 & 20 & 21 & 22 & 43 & 22 \\
\hline & BuzzFeed & 10 & & 21 & 18 & & -12 \\
\hline & Media por país & 23 & 46 & 52 & 27 & 44 & 7 \\
\hline \multirow{6}{*}{ 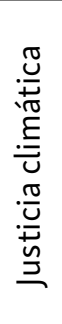 } & Periódico dcha. & 10 & 61 & 63 & 10 & 24 & -12 \\
\hline & Periódico izda. & 47 & 42 & 65 & 23 & 23 & -21 \\
\hline & Huffington Post & 33 & 64 & 48 & 30 & 35 & -9 \\
\hline & Vice & 60 & 20 & 71 & 56 & 14 & -38 \\
\hline & BuzzFeed & 0 & & 25 & 39 & & -16 \\
\hline & Media por país & 30 & 52 & 53 & 34 & 27 & -15 \\
\hline
\end{tabular}

Tabla 3. Distribución de temas por medio, país e ideología (\%)

Fuente: Elaboración propia con datos extraídos de Painter et al., 2017. 
En otros temas, como negociaciones y contexto científico, hay diferencias relevantes entre medios equivalentes de España y los demás países. Prescindiendo de los nativos digitales, podemos aportar el siguiente análisis: el diario español $E l$ País ha publicado más sobre negociaciones que el resto de los periódicos de izquierda de la muestra. También se da la circunstancia de que quienes más abordaron las negociaciones de la cumbre fueron los medios de derechas The Telegraph (Reino Unido) y Frankfurter Allgemeine Zeitung (Alemania). Son también los dos periódicos que ofrecieron más contexto científico (en general, este es aportado en mayor medida por los periódicos de derecha que de izquierda), y tan solo The Guardian (Reino Unido) prestó más atención que ellos al tema de la justicia climática. No obstante, la introducción de la incertidumbre en relación con el cambio climático es más frecuente en los periódicos de derecha que de izquierda.

\section{Fuentes}

Tras contabilizar los distintos tipos de fuentes citadas en los 527 artículos de la muestra (tabla 4), destaca que los dos países de la esfera anglosajona encabezan el número de fuentes citadas, mientras que entre los tres países de la Europa continental Francia aporta mayor número de fuentes, y España se posiciona en último lugar.

Las fuentes que figuran en más artículos corresponden a grupos de interés, destacando especialmente en Estados Unidos y Reino Unido. A continuación, aparecen los políticos nacionales y extranjeros. Sin embargo, en el caso de los medios españoles no se reproduce esta tendencia general. Los políticos extranjeros se citan con más frecuencia $(4,43 \%)$ que los representantes de grupos de interés $(2,9 \%)$ y que los políticos nacionales $(2,21 \%)$. De este modo, España es el país que más se apoya en las fuentes políticas extranjeras y el que menos en las nacionales.

Los científicos son fuentes destacadas en los artículos relacionados con la cumbre de París, pero las informaciones de los medios españoles les otorgaron menos protagonismo que el resto de los medios del ámbito internacional. A diferencia de los medios anglosajones, los europeos dieron poca cabida a la llamada vox populi (ciudadanos), y también en este tipo de citas España se queda a la cola.

Los representantes de la Convención Marco de las Naciones Unidas sobre el Cambio Climático son más relevantes para los medios del país anfitrión de la cumbre que para el resto, lo que simplemente puede estar indicando un acceso más directo de los medios franceses a esas fuentes. Las fuentes menos citadas son los líderes religiosos. En el conjunto de países de la muestra, España es el que más artículos incluye en este ámbito. 


\begin{tabular}{ccccccc} 
& España & Alemania & Francia & $\begin{array}{c}\text { Reino } \\
\text { Unido }\end{array}$ & EE.UU. & TOTAL \\
\hline Político nacional & 2,21 & 3,32 & 3,18 & 3,73 & 4,70 & 17,15 \\
\hline Político extranjero & 4,43 & 3,32 & 1,80 & 3,46 & 3,60 & 16,60 \\
\hline $\begin{array}{c}\text { Representante Convención } \\
\text { Marco Naciones Unidas }\end{array}$ & 0,83 & 0,55 & 2,21 & 0,69 & 0,00 & 4,29 \\
\hline $\begin{array}{c}\text { Representante } \\
\text { organismo internacional }\end{array}$ & 0,69 & 0,83 & 0,83 & 1,24 & 1,66 & 5,26 \\
\hline Científico & 1,38 & 2,90 & 1,94 & 2,90 & 2,49 & 11,62 \\
\hline $\begin{array}{c}\text { Representante de } \\
\text { grupo de interés }\end{array}$ & 2,90 & 2,63 & 3,60 & 5,26 & 5,39 & 19,78 \\
\hline Empresario & 0,97 & 1,24 & 1,24 & 1,38 & 1,24 & 6,09 \\
\hline Ciudadano & 0,28 & 0,83 & 0,83 & 2,49 & 3,18 & 7,61 \\
\hline Líder religioso & 0,83 & 0,28 & 0,41 & 0,28 & 0,69 & 2,49 \\
\hline Otros & 1,38 & 0,55 & 3,04 & 1,38 & 2,77 & 9,13 \\
\hline TOTAL & 15,91 & 16,46 & 19,09 & 22,82 & 25,73 & 100 \\
\hline
\end{tabular}

Tabla 4. Fuentes (\%)

Fuente: Elaboración propia.

\section{DISCUSIÓN}

La discusión que se presenta a continuación confronta los resultados del análisis de contenido con la propuesta de la existencia de tres bloques de países en relación con la cobertura del cambio climático (Painter \& Schäfer, 2018).

\section{Volumen de cobertura}

Los resultados de este trabajo refrendan el análisis comparativo previo de Schmidt et al. (2013), que sitúa el nivel de atención hacia el cambio climático en Reino Unido y Estados Unidos por encima de la media de un conjunto de 27 países. En el caso de Francia, se sitúa en la media, y Alemania y España por debajo de la media. Estos datos podrían interpretarse como una ratificación de los bloques geográficos de cobertura mediática del cambio climático (anglosajón y europeo continental) señalados por Painter y Schäfer (2018). No obstante, las marcadas diferencias en el volumen de cobertura entre los países de la Europa continental incluidos en el estudio (Francia, Alemania y España) indican que, en realidad, no puede hablarse de un bloque homogéneo. Asimismo, en este caso es España la que está por delante de Alemania en número de artículos, cuando trabajos como el Schmidt et al. (2013) han observado resultados inversos. En este sentido, no se cumple lo indicado por Barkemeyer et al. (2017) respecto de que los medios de comunicación de los países con más desarrollo legislativo sobre el cambio climático 
y mejores datos económicos ofrecen una mayor cobertura. Si este criterio fuera acertado, Alemania sería el país europeo con más volumen de información, pero los resultados lo sitúan tras Francia y España.

Del mismo modo, durante el periodo estudiado, el volumen de cobertura en los medios tradicionales españoles no sigue la tendencia internacional (sobre todo con respecto al bloque anglosajón), según la cual la atención de los medios sobre el cambio climático varía sensiblemente en función del alineamiento político de cada medio, con mayor cobertura por parte de los medios de izquierdas (Carvalho, 2007; Dirikx \& Gelders, 2010; Dotson et al., 2012). En España - al igual que en Alemania, pero a diferencia de Francia-, el cambio climático parece ser un asunto asentado en la agenda de los medios tradicionales, independientemente de su línea editorial. Este dato es relevante, ya que supone un cambio de tendencia respecto de la cobertura de otros hitos anteriores sobre el cambio climático. Cabe recordar que, en diciembre de 2009, coincidiendo con la cumbre de Copenhague, que marcó el máximo histórico de cobertura en los periódicos españoles, El País publicó 281 artículos, frente a 156 en El Mundo. En general, hasta aquel momento El País publicó más artículos sobre el cambio climático que El Mundo. Sin embargo, a partir de esa fecha, la cobertura mensual de El Mundo ha sido generalmente más extensa (Fernández-Reyes, 2018).

En nuestro estudio, los datos de cobertura en Alemania sitúan a los medios tradicionales de este país en una situación similar a la de España. Sin embargo, los datos que aportan Boykoff y sus colegas (2018) indican que sí se sigue manteniendo la tendencia a que los medios de la derecha (en este caso, el periódico Die Tageszeitung) otorguen una cobertura menor al cambio climático. Por lo tanto, España vuelve a marcar diferencias respecto de otros países del bloque europeo continental.

Los medios nativos digitales españoles siguen la tendencia internacional de publicar menos artículos sobre la cumbre de París que los medios tradicionales, a excepción de la edición estadounidense y francesa de Huffington Post. En primera instancia, este hecho debe interpretarse como un indicador de que los responsables de estos medios consideran que el cambio climático no es un asunto de gran interés para el público al que se dirigen, mayoritariamente joven.

Dado que los tres medios nativos digitales analizados son empresas con implantación internacional, los tres tenían fácil acceso a artículos sobre la COP21 producidos por las filiales de sus empresas en otros países. Sin embargo, en Vice se limitaron a traducir y publicar aquellos contenidos producidos por su delegación en París que consideraron de interés para su audiencia. Por lo tanto, del escaso número de artículos publicados (7) se deduce que el asunto fue considerado poco atractivo. El interés fue todavía menor por parte de Buzzfeed, que no publicó ningún 
artículo sobre la cumbre. En el caso de Huffington Post, el número de artículos publicados (37) fue superior al de los otros medios nativos digitales españoles, pero muy inferior al de otras ediciones de este medio, como las de Estados Unidos y Reino Unido. Por ende, en este aspecto, los datos de cobertura de los medios nativos digitales sitúan a España dentro del bloque de Europa continental donde el interés por el cambio climático es inferior al del bloque anglosajón.

\section{Temas}

Los resultados del análisis no permiten establecer tendencias claras en cada uno de los bloques geográficos, ya que los porcentajes de frecuencias varían sensiblemente de unos países a otros y de unos medios a otros. Cabe destacar que, frente al énfasis en las temáticas de desastre/catástrofe que detectaron estudios previos (Painter, 2013), estos resultados apuntan a cierto equilibrio entre el enfoque catastrófico y el de oportunidad.

En general, la cobertura realizada en España se distingue del resto por una menor presencia del tema justicia climática, pero serían necesarios datos adicionales para analizar las causas de esta diferencia respecto de la información ofrecida por los otros países. Lo que sí se ha detectado es un tratamiento temático diferenciado en El País con respecto a los otros periódicos de la izquierda: es el segundo medio de izquierda que prestó mayor atención a las oportunidades del cambio climático (por ejemplo, con el artículo del director de innovación de Green Business Spain, Luis Morales Carballo, titulado "Hacia el final de una economía basada en las energías fósiles") y el único de esta ideología que no destacó en el tema de desastre/catástrofe por encima del periódico nacional conservador.

Por último, aunque los medios de derechas sean prolijos en la publicación de artículos sobre las negociaciones del clima, se han detectado contenidos críticos al respecto. Por ejemplo, tras el acuerdo de París, en Alemania el Frankfurter Allgemeine Zeitung considera que este tipo de acuerdos colectivos nunca se cumplen y menciona los peligros del pacto climático para la industria alemana. En Reino Unido, el Telegraph se queja de la dureza del acuerdo para el país, y el francés Le Figaro ofrece una entrevista con el economista Rémy Prud'homme, que defiende la industria energética francesa. Por el contrario, en España, El Mundo (derecha) manifiesta lo limitado de las medidas acordadas.

\section{Fuentes}

En líneas generales, se aprecian algunas similitudes en el uso de las fuentes en los países anglófonos, que colocan en primer lugar a los representantes de grupos de interés, seguidos por políticos nacionales y extranjeros, con proporciones parecidas. En este sentido, el bloque anglosajón no se ajusta a los resultados de otros estudios que contabilizan (por delante de los grupos de interés) a las 
voces políticas y gubernamentales (Eide \& Kunelius, 2010; Comfort et al., 2020). Los países de la Europa continental no muestran una tendencia tan uniforme en las fuentes empleadas, por lo que en este punto no se los puede considerar como un bloque unitario.

Los medios españoles destacan por su escasez en el empleo de fuentes (García Santamaría, 2010). El acceso a las fuentes se relaciona muchas veces de forma directa con la disponibilidad de recursos, por lo que esta puede ser una explicación plausible de por qué, a diferencia de otros, España emplea menos fuentes en sus artículos. Además, los medios españoles utilizan en mayor medida fuentes políticas e internacionales (con frecuencia procedentes de agencias). De este modo, los resultados de nuestra investigación refrendan los de estudios anteriores (Piñuel, 2013).

Por otra parte, el hecho de que España sea el país que más se apoya en las fuentes políticas extranjeras y el que menos en las nacionales podría estar indicando una menor presencia de debate político nacional sobre el cambio climático que en Estados Unidos y otros países europeos. La presencia de citas de líderes religiosos, en el caso español, está en parte ligada al Papa Francisco. La cobertura en los diarios españoles El País y El Mundo de la encíclica Laudato Si ha sido estudiada por Fernández-Reyes (2016), quien señala que ha contribuido a una mayor presencia mediática del cambio climático.

\section{CONCLUSIONES}

Los resultados de este estudio permiten matizar la existencia de los bloques geográficos anglosajón y europeo-continental en la cobertura del cambio climático, señalados en trabajos anteriores (Painter \& Schäfer, 2018), ya que España es notoriamente diferente de otros países de su entorno en cuanto a la cobertura del cambio climático que realizan los medios en línea.

En términos generales, estos bloques se ven aquí refrendados por las tendencias que aparecen en la cobertura, aunque también se constatan diferencias entre los países del bloque de la Europa continental. El diferente volumen de cobertura en Alemania, Francia y España no avala una equiparación entre estos países.

En cuanto a los temas de los artículos, no se aprecian tendencias claras en cada uno de los bloques geográficos. La cobertura realizada en España no presenta grandes diferencias respecto de la de los países del bloque anglosajón o de los otros países del bloque de la Europa continental, con la salvedad de la justicia climática, un asunto que parece estar menos consolidado en la agenda mediática española que en la de otros países, sin que nos consten los motivos. 
La cobertura en línea realizada por los medios españoles es también diferente a la de otros países del bloque de Europa continental, en lo referente al tipo de fuentes utilizadas. En España se constata un mayor peso específico de las fuentes políticas extranjeras frente a las nacionales, lo que sugiere una menor presencia del debate político y social sobre el cambio climático en el ámbito nacional, cuestión que futuras investigaciones podrían abordar.

Nuestro estudio también permite concluir que en 2015 el cambio climático era un tema relativamente consolidado en la agenda informativa, con independencia de la línea editorial de cada medio. A diferencia de otros países, tanto del bloque anglosajón como del de Europa continental, la cobertura realizada en España rompe la tendencia internacional según la cual los medios alineados políticamente a la izquierda publican más artículos sobre el cambio climático que los alineados a la derecha.

Los cinco medios seleccionados en España no son necesariamente una muestra representativa del conjunto de los medios en línea españoles. Sin embargo, la muestra de medios analizados es la que permite lograr el objetivo propuesto, que es comparar la cobertura realizada por los medios en línea españoles con los mismos medios o con otros de similares características de otros países.

Las especificidades detectadas en la cobertura que la prensa española hace del cambio climático nos llevan a formular las siguientes recomendaciones:

- Apostar por una mayor atención mediática, dada la gravedad de los impactos del cambio climático en España y sus implicaciones.

- Ampliar el número y la diversidad de fuentes informativas, para que el cambio climático pase de ser un tema predominantemente político e internacional a un asunto relevante en el contexto nacional, en el que las voces políticas, científicas y de la sociedad civil se escuchen.

- Seguir manteniendo el cambio climático fuera de la polarización ideológica de los medios.

\section{REFERENCIAS}

Anderson, A. (2011). Sources, media, and modes of climate change communication: the role of celebrities. Wiley interdisciplinary reviews: climate change, 2(4), 535-546.

https://doi.org/10.1002/wcc.119

Anderson, A. (2015). News organisation(s) and the production of environmental news. In A. Hansen \& R. Cox (Eds.), The Routledge handbook of environment and communication (pp. 176-185). Abingdon, United Kingdom: Routledge. 
Barkemeyer, R., Figge, F., Hoepner, A., Holt, D., Kraak, J. M., \& Yu, P. S. (2017). Media coverage of climate change: An international comparison. Environment and Planning C: Politics and Space, 35(6), 1029-1054. https://doi.org/10.1177/0263774X16680818

Berelson, B. (1952). Content Analysis in Communication Research. Glencoe: Free Press.

Berglez, P. (2008). What is global journalism? Journalism Studies, 9(6), 845-858. https://doi.org/10.1080/14616700802337727

Berglez, P., Höijer, B., \& Olausson, U. (2009). Individualisation and nationalisation of the climate issue: Two ideological horizons in Swedish news media. In T. Boyce \& J. Lewis (Eds.), Climate Change and the Media (pp. 211-223). New York, NY: Peter Lang.

Blanco-Castilla, E., Quesada, M., \& Teruel Rodríguez, L. (2013). From Kyoto to Durban. Mass media editorial position about climate change. Revista Latina de Comunicación Social, (68), 420-435. https://doi.org/10.4185/RLCS-2013-983en

Boykoff, M. T. \& Boykoff, J. M. (2007). Climate Change and Journalistic Norms. A Case-study of US Mass-media Coverage. Geoforum, 38(6), 1190-1204.

https://doi.org/10.1016/j.geoforum.2007.01.008

Boykoff, M. T., Daly, M., McAllister, L., McNatt, M., Nacu-Schmidt, A., Oonk, D., \& Pearman, O. (2018). German Newspaper Coverage of Climate Change or Global Warming, 2004-2018 April 2018 (data set). https://doi.org/10.25810/RXTV-EB29.1

Brossard, D., Shanahan, J., \& McComas, K. (2004). Are issue-cycles culturally constructed? A comparison of French and American coverage of global climate change. Mass Communication Society, 7(3), 359-377. https://doi.org/10.1207/s15327825mcs0703_6

Carvalho, A. (2007). Ideological Cultures and Media Discourses on Scientific Knowledge: Re-reading News on Climate Change. Public Understanding of Science, 16(2), 223-243. https://doi.org/10.1177/0963662506066775

Carvalho, A. (2005). Representing the politics of the greenhouse effect. Critical Discourse Studies, 2(1), 1-29.

CMNUCC. (1992). Convención Marco de las Naciones Unidas sobre el Cambio Climático (United Nations Framework Convention on Climate Change). Retrieved from http://unfccc.int/resource/docs/convkp/convsp.pdf

Comfort, S. E., Tandoc, E., \& Gruszczynski, M. (2020). Who is heard in climate change journalism? Sourcing patterns in climate change news in China, India, Singapore, and Thailand. Climatic Change, 158(3), 327-343. https://doi.org/10.1007/s10584-019-02597-1

Comscore. (2015). Top 20 España Junio de 2015 (Top 20 Spain June 2015). Retrieved from http://www.comscore.com/esl/Prensa-y-Eventos/Market-Rankings/Top-20-Espana-Junio-2015

Corbett, J. B., Young, L. E., \& Davis, B. L. (2009). Teoría del conflicto e información sobre el cambio climático: interacción entre medios, ciencia, política, industria y audiencias (Theory of conflict and information: interaction among science, politics, industry and audiences). Infoamérica: Iberoamerican Communication Review, (1), 5-23. Retrieved from https://www.infoamerica.org/icr/n01/infoamerica01_corbett.pdf

Corbett, J. B. \& Durfee, J. L. (2004). Testing public (un)certainty of science - media representations of global warming. Science Communication, 26(2), 129-151.

https://doi.org/10.1177/1075547004270234 
De Lara, A. (2013). Las Fuentes Periodísticas y la Información sobre el Cambio Climático en los Medios Españoles: Televisión y Prensa (Journalistic sources and information on climate change in Spanish media: television and press). In B. León (Ed.), El Periodismo ante el Cambio Climático. Nuevas Perspectivas y Retos (Journalism faces climate change: new perspectives and challenges) (pp. 71-84). Barcelona, Spain: Editorial UOC.

Dirikx, A. \& Gelders, D. (2010). To Frame is to Explain: A Deductive Frame-Analysis of Dutch and French Climate Change Coverage during the Annual UN Conferences of the Parties. Public Understanding of Science, 19(6), 732-742. https://doi.org/10.1177/0963662509352044

Dotson, D. M., Jacobson, S. K., Kaid, L. L., \& Carlton, J. S. (2012). Media coverage of climate change in Chile: A content analysis of conservative and liberal newspapers. Environmental Communication: A Journal of Nature and Culture, 6(1), 64-81. https://doi.org/10.1080/17524032.2011.642078

Eide, E. \& Kunelius, R. (2010). Domesticating Global Moments. A transnational study on the coverage of the Bali and Copenhagen Climate Summits. In E. Eide, R. Kunelius, \& V. Kumpu (Eds.), Global Climate-local journalisms (pp. 11-50). Bochum, Germany: Projekt verlag.

European Environment Agency. (2017). Climate change, impacts and vulnerability in Europe 2016. Retrieved from https://www.eea.europa.eu/publications/climate-change-impactsand-vulnerability-2016

Fernández-Reyes, R. (2016). La Encíclica Laudato Si en El País y en El Mundo (The Laudato Si Encyclical in El País and El Mundo). Intexto, (36), 183-199. https://doi.org/10.19132/1807-8583201636.183-199

Fernández-Reyes, R. (2018). Spanish Newspaper Coverage of Climate Change or Global Warming, 2000-2018. Universidad de Sevilla and Center for Science and Technology Policy Research, Cooperative Institute for Research in Environmental Sciences, University of Colorado, Media and Climate Change Observatory Data Sets. https://doi.org/10.25810/37f9-1j65

Fernández-Reyes, R. F., Piñuel Raigada, J. L., \& Águila Coghlan, J. C. (2017). Contraste de la cobertura periodística del cambio climático y del calentamiento global en España y en el ámbito internacional: IV-V Informes del IPCC y Bali-Copenhague-París (A contrast between journalistic coverage of climate change and global warming in Spain and internationally). Revista Latina de Comunicación Social, (72), 1165-1185. https://doi.org/10.4185/RLCS-2017-1213

García Santamaría, J. V. (2010). Crisis del periodismo de fuentes. Las prácticas del periodismo en España en el accidente de Spanair (The crisis of informational sources. The journalism practice in Spain in Spanair's accident). Revista Latina de Comunicación Social, (65), 516-537. https://doi.org/10.4185/RLCS-65-2010-916-516-537

Gavin, N. T. (2009). Addressing climate change: A media perspective. Environmental Politics, 18(5), 765-780. https://doi.org/10.1080/09644010903157081

Harrison, K. \& Sundstrom, L. M. (2007). The Comparative Politics of Climate Change. Global Environmental Politics, 7(4), 1-18. https://doi.org/10.1162/glep.2007.7.4.1 
IAB. (2015, January 11). Los medios de comunicación online son los soportes digitales que mayor credibilidad tienen (Online media are the digital media that have the greatest credibility). IAB España. Retrieved from https://iabspain.es/los-medios-decomunicacion-online-son-los-soportes-digitales-que-mayor-credibilidad-tienen

Kunelius, R. \& Eide, E. (2012). Moment of Hope, Mode of Realism: On the Dynamics of a Transnational Journalistic Field During UN Climate Change Summits. International Journal of Communication, 6, 266-285. Retrieved from https://ijoc.org/index.php/ijoc/article/view/1405

León, B. (2014). El Cambio Climático en los Medios: Una Visión Pluridimensional (Climate change in the media: a pluridimensional view). In B. León (Ed.), Periodismo, Medios de Comunicación y Cambio Climático (Journalism, Media and Climate Change) (pp. 11-27). Salamanca, Spain: Comunicación Social.

León, B. \& de Lara, A. (2013). Ciencia y cambio climático. Estudio de la cobertura del cambio climático en la prensa española (Science and climate change. A study of climate change coverage in the Spanish press). In R. Fernández-Reyes (Ed.), Medios de Comunicación y Cambio Climático (Media and Climate Change) (pp. 91-104). Sevilla, Spain: Fénix.

Lockwood, A. (2009). Preparations for a Post-Kyoto Media Coverage of UK Climate Policy. In T. Boyce \& J. Lewis (Eds.), Climate Change and the Media (pp. 186-199). Nueva York: Peter Lang.

Lopera, E. \& Moreno, C. (2014). The uncertainties of climate change in Spanish daily newspapers: Content analysis of press coverage from 2000 to 2010. Journal of Science Communication,13(01), A2. https://doi.org/10.22323/2.13010202

McCright, A. \& Dunlap, R. (2003). Defeating Kyoto: The conservative Movement's impact on US climate change policy. Social Problems, 50(3), 384-373.

https://doi.org/10.1525/sp.2003.50.3.348

Media and climate Change Observatory, University of Colorado (2021). MeCCO Media and climate change observatory. Retrieved from https://sciencepolicy.colorado.edu/icecaps/ research/media_coverage/index.html

Negredo, S. (2017). Los Sitios Web de Periódicos Afrontan la Creciente Competencia de Televisiones y Nativos Digitales (Journalistic websites face increasing competition of television channels and native digital media). Digital News Report. Retrieved from https://www.digitalnewsreport.es/2016/los-digitales-de-periodicos-afrontan-lacreciente-competencia-de-televisiones-y-nativos-digitales/

Painter, J. (2013). Climate change in the media: reporting risk and uncertainty. Oxford, United Kingdom: Reuters Institute for the Study of Journalism.

Painter, J. (2017). Disaster, risk or opportunity? A ten-country comparison of themes in coverage of the IPCC AR5. In R. Kunelius, E. Eide, M. Tegelberg, \& D. Yagodin (Eds.), Media and Global Climate Knowledge (pp. 109-128). New York, NY: Palgrave Macmillan.

Painter, J. \& Ashe, T. (2012). Cross-national comparison of the presence of climate scepticism in the print media in six countries, 2007-10. Environmental research letters, 7(4), 044005. https://doi.org/10.1088/1748-9326/7/4/044005

Painter, J., Erviti, M. C., Fletcher, R., Howarth, C., Kristiansen, S., Bienvenido, L., ... \& Schäfer, M. S. (2017). Something old, something new: Digital media and the coverage of climate change. Oxford, United Kingdom: Reuters Institute for the Study of Journalism. 
Painter, J. \& Schäfer, M. S. (2018). Global similarities and persistent differences: a survey of comparative studies on climate change communication. Climate change in the media. New York, NY: Peter Lang.

Petersen, A. M., Vincent, E. M., \& Westerling, A. L. (2019). Discrepancy in scientific authority and media visibility of climate change scientists and contrarians. Nature communications, 10 (1), 1-14. https://doi.org/10.1038/s41467-019-09959-4

Piñuel, J. L. (2013). El Discurso Hegemónico de los Media sobre el Cambio Climático (Riesgo, Incertidumbre y Conflicto) y Estrategias de Intervención (The hegemonic media discourse on climate change -risk, uncertainty and conflict- and intervention strategies). In R. Fernández-Reyes (Ed.), Medios de Comunicación y Cambio Climático (Media and Climate Change) (pp. 27-44). Sevilla, Spain: Fénix.

Rubio, J. (2019, February 3). La nueva prensa digital se enfrenta a su primera gran crisis (The new digital press faces its first large crisis). El País. Retrieved from https://verne.elpais. com/verne/2019/02/01/articulo/1549038378_127709.html

Schmidt, A., Ana Ivanova, A., \& Mike S. Schäfer, M. S. (2013). Media Attention for Climate Change around the World: A Comparative Analysis of Newspaper Coverage in 27 Countries. Global Environmental Change, 23(5), 1233-1248. https://doi.org/10.1016/j.gloenvcha.2013.07.020

Schmid-Petri, H. (2017). Do conservative media provide a forum for skeptical voices? The link between ideology and the coverage of climate change in British, German, and Swiss newspapers. Environmental communication, 11(4), 554-567. https://doi.org/10.1080/17524032.2017.1280518

Weart, S. (2021, February). The Public and Climate Change, [online]. Website The Discovery of Global Warming. Retrieved from https://history.aip.org/climate/public.htm

Weingart, P., Engels, A., \& Pansegrau, P. (2000). Risks of communication: Discourses on climate change in science, politics, and the mass media. Public Understanding of Science, 9(3), 261-283. https://doi.org/10.1088/0963-6625/9/3/304

Wigley, T. M. L. (1999). The science of climate change: global and US perspectives. Washington, DC: Pew Center on Global Climate Change.

Zehr, S. C. (2000). Public representations of scientific uncertainty about global climate change. Public Understanding of Science, 9(2), 85-103. https://doi.org/10.1088/0963-6625/9/2/301

\section{AGRADECIMIENTOS}

Este trabajo forma parte de un proyecto liderado por el Dr. James Painter, investigador asociado al Reuters Institute for the Study of Journalism de la Universidad de Oxford, y apoyado por Google y Digital News Iniciative, la Fundación Europea para el Clima y Energy Foundation. 


\section{SOBRE LOS AUTORES}

MARÍA CARMEN ERVITI ILUNDÁIN, Doctora en Comunicación y profesora de Comunicación Empresarial en ISSA-Escuela de Gestión Aplicada de la Universidad de Navarra (España). Es miembro del Grupo de Investigación en Comunicación de la Ciencia de esa Universidad. Su investigación se centra en el estudio del periodismo y la comunicación científica.

iD https://orcid.org/0000-0003-1588-0955

BIENVENido león, Profesor Titular de Periodismo Científico y Producción Televisiva en la Facultad de Comunicación de la Universidad de Navarra (España). Ha publicado más de 80 artículos revisados por pares y 23 libros como autor o editor. Es Fundador y Coordinador del Grupo de Investigación en Comunicación de la Ciencia de la Universidad de Navarra.

(iD) http://orcid.org/0000-0001-8556-9367 